\title{
Driving Scheme Using MIS Photosensor for Luminance Control of AMOLED Pixel
}

\author{
Yuri Vygranenko, Miguel Fernandes, Andrei Sazonov, Senior Member, IEEE, and Manuela Vieira
}

\begin{abstract}
This paper presents a new driving scheme utilizing an in-pixel metal-insulator-semiconductor (MIS) photosensor for luminance control of active-matrix organic light-emitting diode (AMOLED) pixel. The proposed 3-TFT circuit is controlled by an external driver performing the signal readout, processing, and programming operations according to a luminance adjusting algorithm. To maintain the fabrication simplicity, the embedded MIS photosensor shares the same layer stack with pixel TFTs. Performance characteristics of the MIS structure with a nc-Si : $\mathrm{H} / a-\mathrm{Si}: \mathrm{H}$ bilayer absorber were measured and analyzed to prove the concept. The observed transient dark current is associated with charge trapping at the insulator-semiconductor interface that can be largely eliminated by adjusting the bias voltage during the refresh cycle. Other factors limiting the dynamic range and external quantum efficiency are also determined and verified using a small-signal model of the device. Experimental results demonstrate the feasibility of the MIS photosensor for the discussed driving scheme.
\end{abstract}

Index Terms-Amorphous materials, displays, photodetectors, thin films.

\section{INTRODUCTION}

I $\mathrm{N}$ RECENT advances in display technology the active matrix organic light emitting diode (AMOLED) panel is a strong candidate to replace the widespread LCDs due to attributes such as wide viewing angle, high contrast ratio, low power consumption, thin and lightweight construction, and possibility of using a flexible substrate [1]. It is attractive to use conventional hydrogenated amorphous silicon (a-Si:H) backplane technology for fabrication of low-cost large-area AMOLED displays for TV market. However, the a-Si:H pixel circuitry suffers from temporal instability caused by the threshold voltage shift $\left(V_{\mathrm{th}}\right.$-shift) of the driving thin film transistor (TFT) under prolonged bias stress [2]. Although, several correction schemes for the threshold voltage drift have been proposed, the drawbacks are the complexity of driving circuitry, high power consumption, and long programming time due to additional operating cycles [3]-[5]. Another significant issue is luminance non-uniformity and degradation related to the OLED material itself. This problem has to be addressed either by improving the OLED material properties or by adopting the pixel architecture with closed loop luminance control [6]. The control of pixel luminance can be achieved by measuring

Manuscript received December 07, 2012; revised February 22, 2013; accepted March 03, 2013. Date of publication March 29, 2013; date of current version August 07, 2013. This work was supported in part by the Portuguese Foundation of Science and Technology through the PIDDAC Program funds and research Project PTDC/EEA-ELC/115577/2009.

Y. Vygranenko, M. Fernandes, and M. Vieira are with the Department of Electronics, Telecommunication, and Computer Engineering, ISEL, Lisbon, 1959-007 Portugal (e-mail: yvygranenko@deetc.isel.ipl.pt; mv@isel.ipl.pt).

A. Sazonov is with the Electrical and Computer Engineering Department, University of Waterloo, Waterloo, ON, N2L 3G1 Canada.

Digital Object Identifier 10.1109/JDT.2013.2252459 the photon flux from the OLED in real time and adopting a feedback control scheme. The optical feedback technique requires a stable and reproducible in-pixel light sensor to control the pixel brightness. Optical feedback pixel driver (OFPD) circuits with phototransistors [7], [8] and photodiodes [9] have been proposed. However, the fabrication of photoconductors or phototransistors with reproducible characteristics across the large-area backplane is a challenging technological task. Moreover, the sensor itself can exhibit luminance instability due to thermal drift of the photoconductive gain. The drawback of OFPDs with photodiodes is a high implementation cost due to higher mask count and additional processing steps.

Targeting stable and low-cost large-area AMOLED backplane with an efficient luminance control, we propose a driving scheme with an in-pixel MIS-type photosensor sharing the same layer stack with pixel TFTs. The pixel circuitry and external signal processing shown here is the starting point of this work. The bias dependences of the transient dark current and photocurrent are presented and analyzed to optimize the operating conditions of MIS sensor. This follows with the spectral response characteristic of the sensor. The measured external quantum efficiency is correlated with thicknesses of semiconductor and insulator layers to validate the proposed device model. Deduced sensor parameters are used in the calculation example of practical display application. Features of the proposed driving scheme are summarized in the conclusion part.

\section{Pixel Circuitry and Signal Processing}

Fig. 1 shows a pixel circuit with a MIS photosensor, a blockdiagram of the external control unit, and timing diagrams for the proposed driving scheme. The pixel circuit includes a conventional 2-T voltage-programmed driver (transistors T1 and $\mathrm{T} 2$, and storage capacitor $C_{s}$ ), and an additional switching transistor T3 connected between the data line and the MIS photosensor. The MIS photosensor and OLED can be integrated vertically to provide an efficient optical coupling, or, in the case of bottom-emission AMOLED, an optical waveguide has to be implemented between these components.

The control unit comprises a microcontroller with integrated analog-to-digital (ADC) and digital-to-analog (DAC) converters, and a charge amplifier. Switches S1 and S2 are used to configure the unit for pixel programming or charge readout from the sensor.

In the pixel programming period the input video signal is digitized by $\mathrm{ADC} 1$ and the microcontroller calculates the programming voltage for the 2-T driver using the updatable model parameters for the selected pixel. This voltage is then outputted by the DAC and applied to the data line to charge the storage capacitor $C_{s}$ while the voltage level of gate line $\mathrm{A}$ is high. 


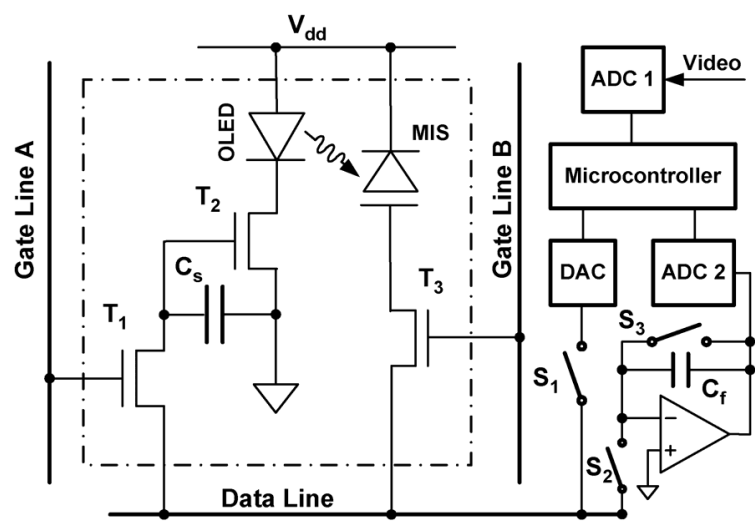

(a)

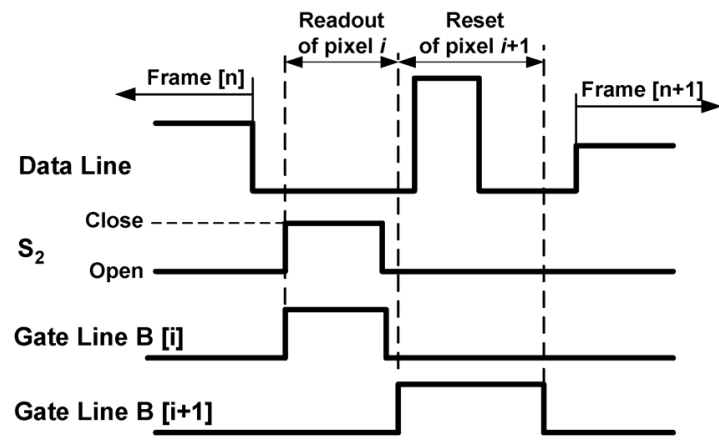

(b)

Fig. 1. (a) Pixel circuit with the external driving electronics and (b) timing diagrams.

The sensing routine is performed during the display operation to continuously refresh the model parameter for each pixel. The sensor works in the integration mode accumulating the lightinduced charge during one frame period. The charge readout and sensor reset are performed within the vertical blanking interval as shown in the timing diagram in Fig. 1(b). Here, when the voltage level of gate line $\mathrm{B}$ in row $i$ becomes high and switch S2 is in the closed state, the accumulated charge from the sensor representing the actual pixel brightness is readout, through switching transistor T3 and the data line, by the charge amplifier. Then, the signal is transformed into digital form by $\mathrm{ADC} 2$ for further processing by the microcontroller. For resetting, when the switch $\mathrm{S} 1$ is closed and gate line $\mathrm{B}$ in row $i+1$ is set to high level, a voltage pulse generated by the DAC is applied to the anode electrode of the sensor.

In the above described sensing routine, the pixel sensing rate is limited for presentation simplicity to 1 row per frame. At this rate, the total acquisition time is to be $9 \mathrm{~s}$ for a full HD display at a refresh rate of $120 \mathrm{~Hz}$. For faster acquisition, the sensing rate should be increased by optimizing the parameters of both sensor and switching TFT that limits the speed of readout and reset operations. For instance, targeting a charge transfer efficiency of $99.3 \%$, the required interval of 5 time constants is $15 \mu \mathrm{s}$ for $100 \times 100 \mu \mathrm{m}^{2}$ sensor at $15 \mathrm{nF} / \mathrm{cm}^{2}$ insulator-semiconductor stack capacitance, and $2 \mathrm{M} \Omega$ on-resistance of the switching TFT. Considering $1080 \mathrm{p}$ video format for HDTV, at a vertical blanking interval of $330 \mu \mathrm{s}$ and a readout-reset time of $33 \mu \mathrm{s}$, the sensing rate can be increased up to 10 rows/frame yielding total acquisition time of $0.9 \mathrm{~s}$.

The luminance adjusting algorithm considered below is similar to the calibration routine compensating the initial luminance deviation of OLEDs in polycrystalline silicon TFT backplanes [10]. We also assume that there is no sensor degradation and all sensors are initially calibrated to compensate the sensitivity nonuniformity due to technological imperfections. The photon flux $\Phi$ directed from the OLED to the MIS photosensor is

$$
\Phi=k \cdot \Phi_{\mathrm{OLED}}=k \cdot \eta_{\mathrm{OLED}} \cdot \frac{I_{\mathrm{OLED}}}{e_{o}}
$$

where $k$ is the optical coupling coefficient, $\Phi_{\text {OLED }}$ the photon flux emitted by the OLED, $\eta_{\text {OLED }}$ the external quantum efficiency of the OLED, $e_{o}$ the elementary charge, and $I_{\text {OLED }}$ the driving current. For $2 \mathrm{~T}$ driver in Fig. 1(a), the output current is

$$
I_{\mathrm{OLED}}=\frac{\mu_{\mathrm{FE}} C_{0 x} W}{2 L}\left(V_{P}-V_{\mathrm{th}}\right)^{2}
$$

where $V_{P}$ is the programming voltage applied to the gate of T2, and the driving transistor parameters $\mu_{\mathrm{FE}}, C, W, L$ and $V_{\mathrm{th}}$ are the mobility, the gate capacitance per unit area, the channel width, the channel length, and the threshold voltage, respectively. Combining (1) and (2), the programming voltage can be defined as

$$
\begin{aligned}
V_{P} & =V_{\mathrm{th}}+B \sqrt{\frac{\Phi}{k}} \\
B & =\sqrt{\frac{2 e_{o} L}{\eta_{\mathrm{OLED}} \mu_{\mathrm{FE}} C_{0 x} W}} .
\end{aligned}
$$

Parameters $V_{\mathrm{th}}$ and $B$ can be determined performing at least two sensing operations at different luminance levels:

$$
\begin{aligned}
V_{\mathrm{th}} & =\frac{V_{P 2} \sqrt{\Phi_{1}}-V_{P 1} \sqrt{\Phi_{2}}}{\sqrt{\Phi_{1}}-\sqrt{\Phi_{2}}} \\
B & =\frac{\sqrt{k}\left(V_{P 1}-V_{P 2}\right)}{\sqrt{\Phi_{1}}-\sqrt{\Phi_{2}}} .
\end{aligned}
$$

The programming voltage can be expresses as

$$
V_{P}=V_{\mathrm{th}}+B \sqrt{\Phi_{\mathrm{OLED}}^{\max } \frac{\text { data }}{n}}
$$

where $\Phi_{\mathrm{OLED}}^{\max }$ is the maximum target photon flux of the OLED, $n$ the total number of gray levels, and data the selected gray level from the input video data.

The proposed method provides a full compensation of brightness non-uniformity during display operation that may be caused by various factors such as the threshold voltage shift of driving transistor, temperature instability of pixel circuit, or any change in the current-voltage characteristics or emission efficiency of the OLED element.

\section{MIS Photosensor Performance}

To analyze the performance characteristics of MIS photosensor, we have fabricated a test structure using the deposition process for back channel passivated nc-Si : H/a - Si : H TFTs [11]. This micromorph technology is attractive for AMOLED 


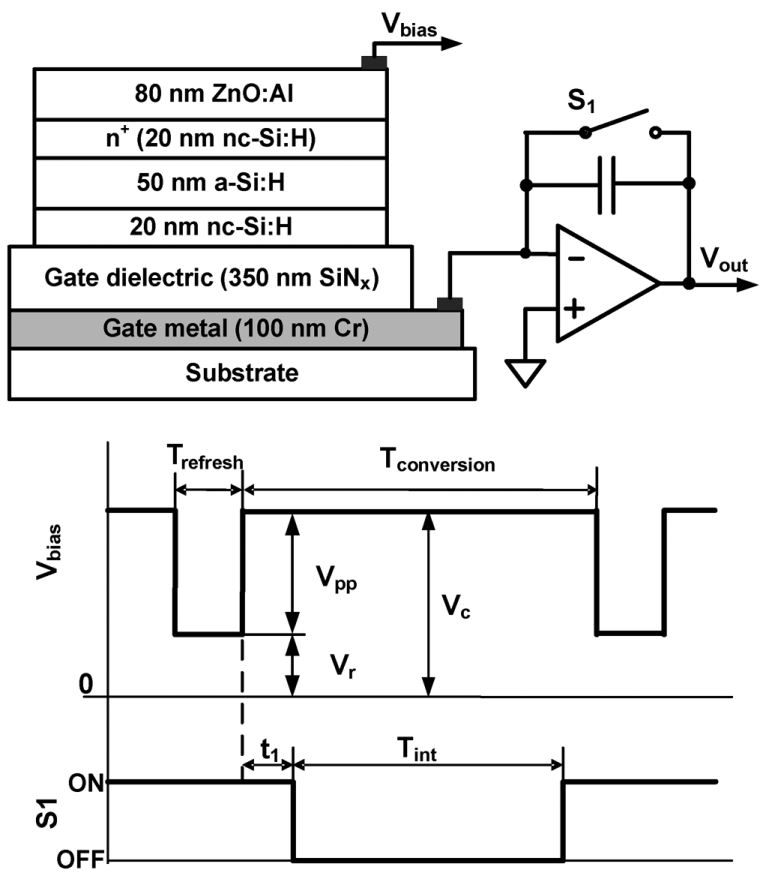

Fig. 2. MIS structure stack and testing circuitry with signal diagrams.

applications due to the enhanced threshold voltage stability of transistors stressed at constant drain currents [12].

Fig. 2 shows a cross-sectional view of the fabricated structure, and a testing circuitry with timing diagrams to illustrate the device operation. Here, the anode of the device remains at ground potential as forced by the charge amplifier, while the biasing pulse of positive polarity is applied to the cathode. The indicated $V_{r}$ and $V_{c}$ are the voltages applied to the cathode during refresh and conversion cycles, respectively, and the peak-topeak pulse amplitude, $V_{p p}$, is a difference between these voltages. During the conversion cycle, the charge amplifier integrates the input current from the MIS structure during the time period $T_{\text {int }}$. A delay $t_{1}=300 \mu \mathrm{s}$ is introduced to avoid detection of the charge induced by the geometrical capacitance of the MIS structure. An active area of the device is $1 \mathrm{~mm}^{2}$ yielding capacitance of about $120 \mathrm{pF}$ that matches the internal capacitor $(100 \mathrm{pF})$ of the used charge amplifier (ACF2101).

When a refresh pulse is periodically applied to the MIS structure, electrons are injected from the $\mathrm{n}^{+}$layer into the intrinsic amorphous silicon and drift to the dielectric interface. Some of these electrons recombine with holes accumulated at the interface while others become trapped in bulk and interface states. During the conversion cycle, the trapped electrons are emitted with a range of emission time constants, leading to a current transient. This dark current component follows a power-law time dependency

$$
I_{\mathrm{dark}}(t)=I_{\tau}\left(\frac{\tau}{t}\right)^{\beta}
$$

where $I_{\tau}$ is the current at time $\tau$, and $\beta$ is the power parameter. The readout charge is given by

$$
Q_{\mathrm{dark}}(t)=\int_{t_{1}}^{t} I_{\mathrm{dark}}(x) d x=\frac{I_{\tau} \tau^{\beta}\left(t^{1-\beta}-t_{1}^{1-\beta}\right)}{1-\beta} .
$$

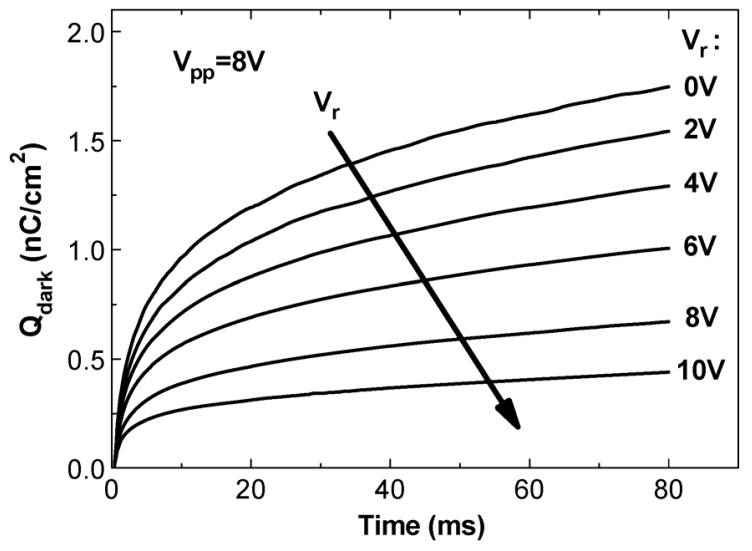

Fig. 3. Dark charge versus time at refresh bias voltages applied to the cathode of the MIS structure. The peak-to-peak bias pulse amplitude is $8 \mathrm{~V}$.

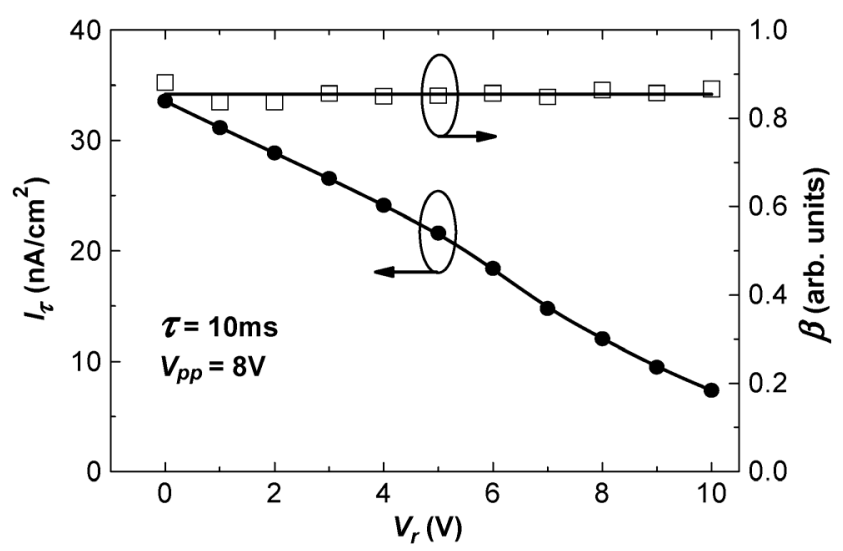

Fig. 4. Variations of $I_{\tau}$ and $\beta$ values with the refresh voltage.

Fig. 3 shows $Q(t)=C_{f} \cdot V_{\text {out }}$ waveforms measured at $V_{p p}=$ $8 \mathrm{~V}$, and $V_{r}=0 \ldots 10 \mathrm{~V}$. Experimental curves were fitted using (9). The deduced $I_{\tau}$ and $\beta$ values are shown in Fig. 4. Values of $\beta$ vary in a narrow range yielding a mean of 0.855 and a standard deviation of 0.005 . However, the amplitude of the transient dark current $\left(I_{\tau}\right.$ at $\left.\tau=10 \mathrm{~ms}\right)$ decreases with increasing $V_{r}$ significantly. This indicates that the occupation of trap levels at the semiconductor-insulator interface and within undoped layers is determined by the bias voltage during the refresh cycle. Similar behavior has been reported for MIS structures with thick $(\sim 500$ $\mathrm{nm}) \mathrm{a}-\mathrm{Si}: \mathrm{H}$ i-layer [13]. Thus, the dark signal limiting the light detection can be reduced by optimizing the refresh voltage.

The sensor linearity was examined by measuring the output photo-charge as a function of time at steady-state illumination. Fig. 5(a) shows $Q_{\text {light }}(t)$ curves measured at different bias pulse amplitudes in the range from 2 to $10 \mathrm{~V}$. The deduced values of the saturation charge versus $V_{p p}$ are shown in Fig. 5(b). Considering a simplified small-signal model of the MIS structure, which is shown in the inset, the saturation charge is

$$
Q_{\mathrm{sat}}=\frac{C_{i}^{2} \cdot V_{\mathrm{pp}}}{C_{i}+C_{s}}
$$

where $C_{i}$ and $C_{s}$ are the net capacitances of the insulator and semiconductor layers. At high bias voltages, the measured saturation charges are consistent with the calculated curve (dashed 


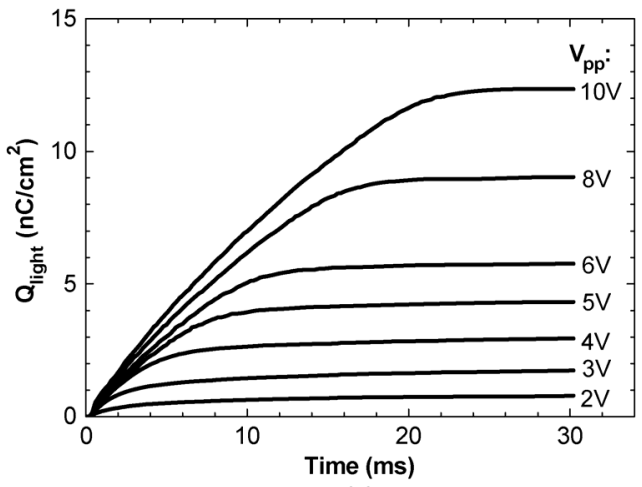

(a)

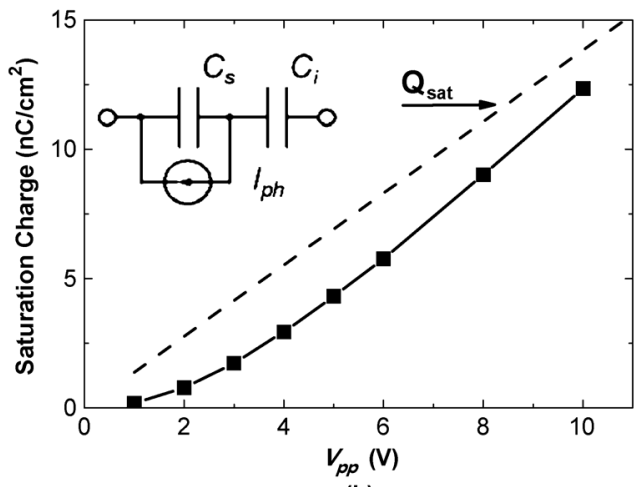

(b)

Fig. 5. (a) Light signal waveforms. (b) Deduced saturation charge density versus the amplitude of bias pulse applied to the MIS structure. The dashed line indicates the values calculated with the small signal model of the MIS structure shown in the inset.

line). The signal saturation is a major factor limiting the dynamic range of the sensor, and it can be improved by reducing the thickness of the gate dielectric and increasing the thickness of intrinsic layers. The observed nonlinearity below saturation level can be also minimized by optimizing $V_{\mathrm{pp}}$ and compensated by signal processing.

Fig. 6 shows an external quantum efficiency (EQE) spectrum of the MIS photosensor. The EQE reaches a peak value of 5.2\% at a wavelength of $490 \mathrm{~nm}$. This value is significantly lower than the absorption coefficient of the a-Si:H-nc-Si:H layers. The reason for this is that the charge generated in the active region cannot be fully readout through the blocking dielectric layer [14]. The ratio of transferred $\left(Q_{\text {out }}\right)$-to-generated $\left(Q_{\text {sig }}\right)$ charges, $\eta_{Q}$, calculated using the equivalent circuit model of the MIS structure is:

$$
\eta_{Q}=\frac{Q_{\mathrm{out}}}{Q_{\mathrm{sig}}}=\frac{C_{i}}{C_{i}+C_{s}} .
$$

For actual values $C_{i}=14.4 \mathrm{nF} / \mathrm{cm}^{2}$ and $C_{s}=130 \mathrm{nF} / \mathrm{cm}^{2}$ the calculated $\eta_{Q}$ is $10 \%$. The reflection and absorption losses at the $\mathrm{TCO} / \mathrm{n}^{+}$interface, and recombination at the $\mathrm{n}^{+} / \mathrm{i}$-junction are additional factors reducing the EQE. Thus, the sensitivity enhancement can be achieved by increasing $\eta_{Q}$ through the optimization of the dielectric/semiconductor layer thicknesses, and also tailoring the $\mathrm{TCO} / \mathrm{n}+/ \mathrm{i}$ interface.

It should be noted that our structure and fabrication process followed tri-layer TFT fabrication sequence, which is less common in the industry. For more widely used

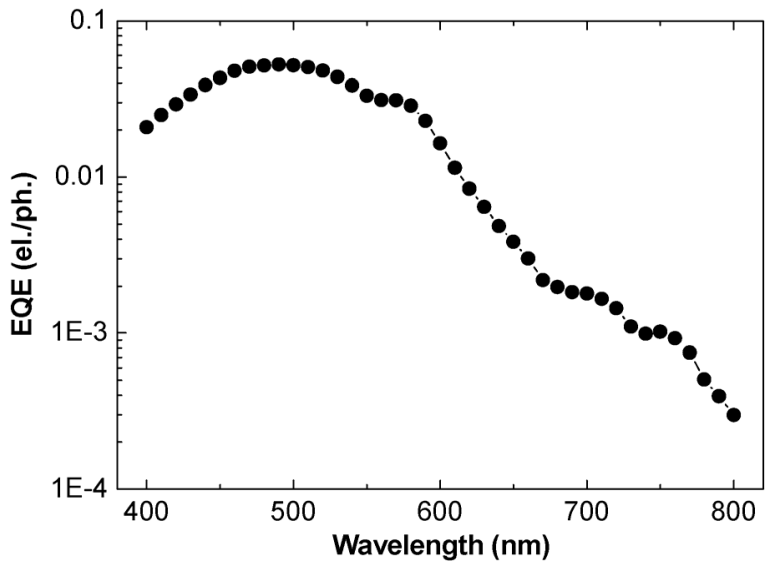

Fig. 6. External quantum efficiency spectrum of the MIS photosensor.

back-channel-etch (BCE) process, the sensor would have thicker a-Si:H layer and thinner gate dielectric that would further improve the EQE and dynamic range.

To demonstrate the feasibility of the sensor for the discussed compensation scheme, let's consider its application for a 32" AMOLED display with resolution of $1920 \times 1080$ pixels. A $369 \times 369 \mu \mathrm{m}^{2}$ pixel comprises three RGB sub-pixels of about equal area. The green sub-pixel contains a $200 \times 100 \mu \mathrm{m}^{2}$ bottom-emission OLED and a $100 \times 100 \mu \mathrm{m}^{2}$ sensor. The signal charge integrated during one frame is

$$
Q_{s}=e_{0} \cdot \eta_{\lambda} \cdot k \cdot \frac{\Phi_{\text {OLED }}}{F}
$$

where $\eta_{\lambda}$ is the external quantum efficiency of the sensor at the emission wavelength, $F$ the refresh rate. The optical coupling coefficient must be carefully defined to avoid the sensor saturation. Setting the upper detection limit to $80 \%$ of the saturation charge, the coefficient can be expressed as

$$
k=\frac{0.8 \cdot Q_{\mathrm{sat}} \cdot A_{s} \cdot F}{e_{0} \cdot \eta_{\lambda} \cdot \Phi_{\mathrm{OLED}}^{\max }} .
$$

Here, parameters $\mathrm{Q}_{\mathrm{sat}}=12 \mathrm{nQ} / \mathrm{cm}^{2}$ at $V_{\mathrm{pp}}=10 \mathrm{~V}$ and $\eta_{\lambda}=0.044$ at $\lambda=530 \mathrm{~nm}$ are obtained from plots in Fig. 5(b) and Fig. 6, respectively. Limiting the green OLED brightness to $300 \mathrm{~cd} / \mathrm{m}^{2}$ that yields $\Phi_{\mathrm{OLED}}^{\max } \approx 3.10^{11}$ photons $/ \mathrm{s}$, and $k=$ 0.055 at $F=120 \mathrm{~Hz}$. At 256 grey levels, the signal charge will be in the range from 3.8 fQ to $960 \mathrm{fQ}$. The minimal signal is higher than the commonly accepted standard of 2000 electrons (or $0.32 \mathrm{fQ}$ ) for noise level in flat panel x-ray imagers, i.e. the existing charge-detection technique can be used for the discussed application.

\section{CONCLUSION}

Although the optical feedback driving technique is able to manage the luminance non-uniformity and OLED efficiency degradation, it introduces other issues such as sensor-induced instability, pixel-design complexity, and high implementation cost. To resolve these issues, we propose a driving scheme utilizing an in-pixel MIS photosensor that is process compatible with the TFT backplane. The presented 3-T 1-C 1-MIS pixel circuit compensates for the degradation of both driving TFT and OLED. Performance characteristics of the MIS structure 
are also analyzed. Measurements of the transient dark current under different biasing conditions reveal that the associated noise component can be largely eliminated by adjusting the bias voltage during the refresh cycle. The light-biased device shows a sub-linear response correlated with biasing conditions. The observed signal saturation is explained using a small-signal model of the MIS structure. Factors limiting the external quantum efficiency are also identified. A calculation example for large-area HD display is also given. These results demonstrate the feasibility of the MIS photosensor for the proposed driving scheme.

\section{ACKNOWLEDGMENT}

The authors are grateful to the Giga-to-Nanoelectronics Centre at the University of Waterloo, Waterloo, Canada, for providing necessary equipment and technical help to carry out this work.

\section{REFERENCES}

[1] G. Gu and S. R. Forest, "Design of flat-panel displays based on organic light-emitting devices," IEEE J. Sel. Top. Quantum Electron., vol. 4, no. 1, pp. 83-99, Jan./Feb. 1998.

[2] Thin-Film Transistors, C. R. Kagan and P. Andry, Eds. New York: Marcel Dekker, 2003.

[3] M. Bagheri, S. J. Ashtiani, and A. Nathan, "Fast voltage-programmed pixel architecture for AMOLED displays," J. Display Technol., vol. 6, no. 5, pp. 191-195, May 2010.

[4] G. Reza Chaji and A. Nathan, "Low-power low-cost voltage-programmed a-Si:H AMOLED display for portable devices," J. Display Technol., vol. 4, no. 2, pp. 233-237, Jun. 2008.

[5] G. R. Chaji, S. Alexander, J. M. Dionne, Y. Azizi, C. Church, J. Hamer, J. Spindler, and A. Nathan, "Stable RGBW AMOLED display with OLED degradation compensation using electrical feedback," in IEEE Solid-State Circuits Conf. Dig. Tech. Papers, 2010, pp. 118-119.

[6] H. Lifka, W. Oepts, N. Bramante, S. Deane, D. Fish, N. Young, A. Steer, D. George, and A. Giraldo, "Optical feedback for AMOLED pixel circuits,” ECS Trans., vol. 3, no. 8, pp. 23-33, Aug. 2006.

[7] N. P. Papadopoulos, A. A. Hatzopoulos, and D. K. Papakostas, "An improved optical feedback pixel driver circuit," IEEE Trans. Electron Devices, vol. 56, no. 2, pp. 229-235, Feb. 2009.

[8] M. H. Kang, J. H. Hur, Y. D. Nam, E. H. Lee, S. H. Kim, and J. Jang, "An optical feedback compensation circuit with a-Si:H thin-film transistors for active matrix organic light emitting diodes," J. Non-Cryst. Solids, vol. 354, pp. 2523-2528, 2008.

[9] D. Fish, N. Young, S. Deane, A. Steer, D. George, A. Giraldo, H. Lifka, O. Gielkens, and W. Oepts, "Optical feedback for AMOLED display compensation using LTPS and a-Si:H technologies," SID Dig., vol. 36, pp. 1340-1343, May 2005.

[10] H.-J. In, K.-H. Oh, O.-K. Kwon, C. H. Hyun, and S.-C. Kim, "A luminance adjusting algorithm for high resolution and high image quality AMOLED displays of mobile phone applications," IEEE Trans. Consumer Electron., vol. 56, pp. 1191-1195, Mar. 2010.

[11] M. R. Esmaeili-Rad, A. Sazonov, and A. Nathan, "Analysis of the off current in nanocrystalline silicon bottom-gate thin-film transistors," $J$. Appl. Phys., vol. 103, p. 074502, Jul. 2008.

[12] M. R. Esmaeili-Rad, A. Sazonov, and A. Nathan, "Absence of defect state creation in nanocrystalline silicon thin film transistors deduced from constant current stress measurements," Appl. Phys. Lett., vol. 91, p. 113511, Nov. 2007.

[13] M. Fernandes, Y. Vygranenko, M. Vieira, G. Heiler, T. Tredwell, and A. Nathan, "Transient current in a-Si:H-based MIS photosensors," in Mater. Res. Soc. Symp. Proc. on Amorphous and Polycrystalline ThinFilm Silicon Science and Technol., A. Flewitt, J. Hou, S. Miyazaki, A. Nathan, and J. Yang, Eds., 2008, vol. 1066, A18-06.
[14] M. Fernandes, Y. Vygranenko, A. Fantoni, R. Martins, and M. Vieira, "Spectral response characterization of a-Si:H-based MIS-type photosensors," Phys. Stat. Solid. (c), vol. 5, pp. 3410-3413, Oct. 2008.

Yuri Vygranenko received the M.S. degree in physics and Ph.D. degree in physics and mathematics from Chernovtsy University, Ukraine, in 1985 and 1997, respectively.

From 1987 to 1999 , he carried out research related to the physics and technology of the IV-VI narrow-gap semiconductors in the Chernovtsy Department of the Institute of Material Science, Academy of Science in Ukraine. From 1999 through 2001, he was at the ISEL, Lisbon, Portugal, where his research activity in the field of solid film device technology was supported by the NATO Science Program for Peace and Security, and National Science Program of Portugal. In 2002, he joined the Electrical and Computer Engineering Department, University of Waterloo, ON, Canada, where he carried out research in the fields of digital radiographic imaging, transparent electronics, and AMOLED displays. He also performed as a senior scientist for joint R\&D program with Ignis Inc., Canada, and Kodak Corp., USA. Since 2005, he has been working as a researcher in the ISEL, Lisbon, Portugal. His research interests include thin-film technology for large-area image sensors, displays, and photovoltaic devices. $\mathrm{He}$ has authored and co-authored over 90 international journal and conference papers and 4 U.S. patents.

Miguel Fernandes was born in Portugal in 1970. He received the degree in physics and materials engineering in 1995, and the Ph.D. degree in materials science and engineering in 2009, all from the Faculty of Sciences and Technology, New University of Lisbon, Lisbon, Portugal.

In 1993, he became researcher in the Center of Excellence for Microelectronics and Optoelectronics Processes-UNINOVA, Lisbon, Portugal. Currently he is a Professor in Electronics, Telecommunication, and Computer Engineering Department of ISEL, Lisbon, Portugal. He is also a researcher in the group of Applied Research in Microelectronic Optoelectronic and Sensors-GIAMOS in the same institution, and in UNINOVA-CTS.

Andrei Sazonov (M'01-SM'06) received the B.Sc. and Ph.D. in electrical engineering from Moscow Institute of Electronic Technology (Technical University), Moscow, Russia, in 1991 and 1997, respectively.

From 1991 to 1993 , he was a Research Engineer at Materials Science Department, Moscow Institute of Electronic Technology, and Research Engineer at Elma Corporation, and Mikron Corporation, Moscow, Russia. From 1995 to 1996, he was a Visiting Research Fellow at the Institute of Physical Electronics, University of Stuttgart, Germany, under a German Academic Exchange Service (DAAD) scholarship. In 1998, he joined Electrical and Computer Engineering Department, University of Waterloo, as a Post-doctoral Research Associate, in 2000 he became an Assistant Professor, and in 2006, an Associate Professor. He was director of University of Waterloo Giga-to-Nanoelectronics (G2N) Centre in 2006-2010. His research interests include thin film technology, amorphous, nanocrystalline, and polycrystalline semiconductors and dielectrics, semiconductor devices and their applications (flat panel displays, thin film transistors, solar cells, radiation sensors, memory devices, RF ID), large area electronics and flexible electronics.

Dr. Sazonov is a recipient of a 2001 Canadian Foundation for Innovation New Opportunities Award, 2002 Ontario Premier's Research Excellence Award, and 2004 IEE Institution Premium.

Manuela Vieira received the M.S. degree in solid state physics-Microelectronic in 1987, and the Ph.D. in semiconductor materials in 1993, both from the New University of Lisbon, Lisbon, Portugal.

She is a full professor in electronics inside the Department of Electronics Telecommunication and Computers, ISEL, Portugal, and the head of a Group in Applied Research in Microelectronic Optoelectronic and Sensors-GIAMOS. She has several hundred scientific papers and 25 years of experience in the field of thin films and devices. Her research activities have been mainly related to the development of optical sensors. 